\title{
Mapping QTLs on chicken chromosome 1 for performance and carcass traits in a broiler $x$ layer cross
}

K. Nones, M. C. Ledur, D. C. Ruy, E. E. Baron, C. M. R. Melo, A. S. A. M. T. Moura,

\author{
E. L. Zanella, D. W. Burt and L. L. Coutinho
}

Keywords : abdominal fat, carcass, growth, organs, quantitative trait loci, selective genotyping 


\section{Summary}

With the objective of mapping quantitative trait loci (QTLs) for performance and carcass traits, an $F_{2}$ chicken population was developed by crossing broiler and layer lines. A total of $2063 F_{2}$ chicks in 21 full-sib families were reared as broilers and slaughtered at 42 days of age. Seventeen performance and carcass traits were measured. Parental (FO) and $F_{1}$ individuals were genotyped with 80 microsatellites from chicken chromosome 1 to select informative markers. Thirty-three informative markers were used for selective genotyping of $F_{2}$ individuals with extreme phenotypes for body weight at 42 days of age (BW42). Based on the regions identified by selective genotyping, seven full-sib families ( $649 \mathrm{~F}_{2}$ chicks) were genotyped with 26 markers. Quantitative trait loci affecting body weight, feed intake, carcass weight, drums and thighs weight and abdominal fat weight were mapped to regions already identified in other populations. Quantitative trait loci for weights of gizzard, liver, lungs, heart and feet, as well as length of intestine, not previously described in the literature were mapped on chromosome 1 . This $F_{2}$ population can be used to identify novel QTLs and constitutes a new resource for studies of genes related to growth and carcass traits in poultry.

\section{Introduction}

Over the last few decades, broiler selection programmes have emphasized fast growth and reduced feed conversion rates. Progress in growth rates has been accompanied by an increase in fat deposition, but a proportional development of heart and lungs has not been observed (Burt 2002). As a result, rapid-growing broilers have increased fat deposition, metabolic disorders and reduced meat quality.

The chicken genome has 38 autosomes of different lengths. Chromosome 1 is the largest, corresponding to $14.9 \%$ of the entire genome (Groenen et al. 2000; International Chicken Genome Sequencing Consortium 2004). Quantitative trait loci (QTL) affecting body weight, growth, feed intake, and weights of breast muscle, thighs, drums, wings and fat deposition were identified on chromosome 1 by Van Kaam et al. (1999a,b), Tatsuda \& Fujinaka (2001), Sewalem et al. (2002), Ikeobi et al. (2002), De Koning et al. (2004), Ikeobi et al. (2004) and Jennen et al. (2004). However, before attempting to identify potential genes and exploiting 
them in animal breeding programmes by marker assisted selection (MAS), confirmation is necessary to verify the existence of QTL observed in an initial genome scan, preferably by using independent populations (Spelman \& Bovenhuis 1998; Marklund et al. 1999).

In this paper, several QTLs for economically important traits for the poultry industry were mapped in a Brazilian $\mathrm{F}_{2}$ chicken population that was derived from a broiler $\mathrm{x}$ layer cross.

\section{Materials and Methods}

\section{Experimental population}

The $F_{2}$ chicken population was developed by crossing a broiler male line (TT) with a layer line (CC). These lines, when reared as broilers, have a fivefold difference in body weight at 41 days of age (Ledur et al. 2000a,b). Seven TT males and seven CC females were crossed to produce $F_{1}$ chickens (TC). Seven F1 TC males were each mated to three unrelated $F_{1}$ TC females to create 21 full-sib families, each with $\sim 98 F_{2}$ chicks. A total of $2063 F_{2}$ offspring from 17 hatches were reared as broilers and slaughtered at 42 days of age. $F_{2}$ chicks were allocated in floor pens with suspended drinkers and tubular feeders. During the experimental period, feed and water were supplied ad libitum.

\section{Phenotypes}

Body weight in grams (g) was recorded at 1, 35 and 42 days of age (BW1, BW35 and BW42, respectively). BW42 corresponded to the weight after $6 \mathrm{~h}$ of fasting and transportation to the slaughter location. Individual caging was employed between 35 and 41 days of age, to allow individual measurement of feed intake (FI35-41), as well as an assessment of feed conversion (FC35-41) and weight gain (WG35-41) between 35 and 41 days of age. Carcasses were eviscerated, and the weights of heart, gizzard, liver, lung and feet, as well as the length of intestine, were recorded. Carcasses were then stored at ) $-4{ }^{\circ} \mathrm{C}$ for $6 \mathrm{~h}$ before dissection, when the weights of carcass (without giblets, feet or head), breast (with bone and skin), drums and thighs, wings, residual carcass (weight of carcass without breast, drums, thighs and wings) and abdominal fat were recorded. 


\section{Genotyping and map construction}

Polymerase chain reactions for each marker were carried out separately in reaction volumes of 25 II. Polymerase chain reaction conditions were as previously described by Nones et al. (2005). Parental (FO) and $F_{1}$ individuals were genotyped with 80 microsatellites to select informative markers. Selective genotyping was employed to identify the informative markers with a nominally significant association (chi-square analysis, $\mathrm{P}<0.10$ ) with BW42 adjusted for hatch and sex (BW42ad). A total of 170 F2 individuals (six to 10 from each of the 21 full-sib families, which represented the top and bottom $4.5 \%$ of the phenotypic distribution with average BW42ad of $1328.5 \pm 127.8$ and $801.5 \pm 93.8$ g, respectively) were genotyped with 33 informative markers.

For QTL mapping, seven full-sib families (with a total of $649 \mathrm{~F}_{2}$ individuals) were genotyped with 12 markers that were associated with BW42ad in the selective genotyping analysis, as well as 14 additional flanking markers. Families were chosen based on the number of informativemarkers. All genotypic data were scored twice and then rechecked for inheritance using CRIMAP (Green et al. 1990). The linkage map was constructedusingCRIMAPas described byNones et al. (2005).

\section{Statistical analysis}

Phenotypic data from the seven selected full-sib families were adjusted for systematic hatch effect and the residuals were used in the QTL analyses. Interval mapping using regression methods was applied to two different genetic models: (1) line-cross analysis following Haley et al. (1994), assuming founder lines to be fixed for different QTL alleles and (2) paternal half-sib model (Knott et al. 1996), making no assumptions about fixation of QTL alleles in the founder 2 lines. The QTL Express software was employed for QTL analyses.

Sex and family effects were tested in a previous analysis and included in the model only if statistically significant. Additional analyses were conducted, including BW35 as a covariate for performance traits (Adj_WG35-41, Adj_FI35-41, Adj_FC35-41) and BW42 as a covariate for carcass traits (Adj_carcass, Adj_wings, Adj_drums and thighs, Adj_residual carcass, Adj_abdominal fat, Adj_feet, Adj_gizzard, Adj_liver, Adj_lungs and Adj_heart, Adj_intestinal 
length). If the test statistics for one QTL exceeded the threshold value, we conducted the following analyses based on conventional F-tests and appropriate degrees of freedom: (a) interaction between QTL effect and sex, (b) interaction between QTL effect and family and (c) model fitting a parent-of-origin effect as described by Knott et al. (1998). When the analysis of any trait presented evidence for one QTL, the presence of two or more QTLS were also tested.

For the half-sib analyses, $F_{2}$ animals from the seven fullsib families were assigned to three paternal half-sib families, i.e. additional genetic relationships within half-sib groups were ignored. A standard F-test was used to test whether the two best QTLs on the chromosome significantly explained $(P<0.05)$ more variance than the single best $Q T L$. Once a QTL was detected (suggestive level) in the acrossfamily analyses, analyses within families were performed, and the test statistics were calculated as an F-ratio for every $\mathrm{CM}$ on the chromosome (De Koning et al. 1999).

\section{Significance thresholds}

Significance thresholds for both line-cross and half-sib analyses were calculated using a permutation test (Churchill \& Doerge 1994). A total of 10000 permutations were computed to determine the empirical distribution of the statistical test under the null hypothesis of no QTL associated with the part of the genome under study. Three significance levels were used: suggestive, $5 \%$ and $1 \%$ genome-wide (Lander \& Kruglyak 1995).

\section{Results and discussion}

\section{Selective genotyping}

Of the 80 markers tested, 33 informative markers were used for selective genotyping and 12 of those were associated with BW42ad (Fig. 1). These 12 markers plus an additional 14 markers employed to reduce marker spacing were used for QTL mapping. 


\section{Line-cross analysis}

Information on QTLs with suggestive and significant linkage is presented in Table 1. Four main QTL regions can be identified on chromosome 1. The first is located between 80 and 100 cM (between LEI0068 and LEI0174), which contains QTLs associated with BW35, BW42, weights of carcass, wings, drums and thighs, residual carcass, Adj_abdominal fat, feet, liver, lungs and heart, intestinal length, and Adj_intestinal length. The second is located between 125 and 139 cM (between ADL0150 and MCW0058), which contains QTLs associated with BW42, FI35-41, carcass weight and breast weight. A third region located between 184 and 200 cM (between LEI0138 and LEI0160) contains QTLS for BW35, residual carcass, abdominal fat and Adj_feet weight. A fourth region located around 332 and 339 cM (between LEI0079 and MCW0145) contains QTLs for BW35, BW42, weights of carcass, wings, breast, feet, gizzard and heart and intestinal length. Additional QTLs were mapped at 28 cM for Adj_wings weight, at 53 cM for lungs weight, at 251 cM for Adj_abdominal fat and at 373 cM for Adj_intestinal length.

Markers LEI0068, MCW0058 and LEI0079 were associated with body weight and several carcass traits, indicating the presence of a common region controlling growth in general. Our results are in agreement with those of Van Kaam et al. $(1999 a, b)$, who found association of marker MCW0058 with body weight at 48 days of age and with feed intake. These authors also found an association of LEI0079 with a QTL for carcass percentage in a cross of two dam broiler lines. In a broiler-layer cross, Sewalem et al. 3(2002) associated LEI0068 and LEI0079 with body weight at 3 and 9 weeks of age respectively. In this same population Ikeobi et al. (2004) found an association of LEI0068 with thigh muscle and drum weights.

In the present study, a QTL for Adj_abdominal fat (94 cM) was mapped between LEI0146 and LEI0174. These markers were also associated with weights of drums and thighs (97 cM), residual carcass (93 cM) and heart (100 cM) (Table 1). Tatsuda \& Fujinaka (2001) and Jennen et al. (2004) also found association of LEI0174 with body weight and abdominal fat weight respectively.

Quantitative trait loci for weights of wings, breast, gizzard, feet, and heart were mapped in regions that coincided with one or two chromosomal regions also mapped for body and carcass weights (Table1). When BW42 was included in the model as covariate, no QTL were 
mapped for these traits in those regions, suggesting that the same QTLs affecting body weight are also influencing the other traits. This supposition is supported by the high genetic correlation between body weight and carcass traits in the current $F_{2}$ population (C.M.R. Melo et al., unpublished data). When BW35 and BW42 were included in the model as covariates for performance and carcass traits, respectively, QTLs for Adj_wings, Adj_abdominal fat, Adj_feet and Adj_intestine length were detected at different positions than the QTLs for BW42, suggesting that other genes may be controlling part of the phenotypic variance of these traits (Table 1).

Most QTLs mapped in this study were of an additive nature with positive effects (Table 1). These results are in agreement with those of Ikeobi et al. (2004). Only QTLs for Adj_abdominal fat, Adj_feet and lungs showed negative additive effects. Dominance effects of QTLs were positive or negative, depending on the trait. Individual QTLs mapped explained $0.81-4.04 \%$ of the total phenotypic variance. It is important to note that when taken together, QTLs on chromosome 1 explain 7\% of phenotypic variance for BW35, $4.2 \%$ of Adj_abdominal fat, $6.3 \%$ of intestine length, $5.7 \%$ of lungs weight and $4.2 \%$ of heart weight. For BW35, BW42, weights of carcass, breast, Adj_abdominal fat, residual carcass, heart, FI35-41, and intestine length, the QTL $x$ family interaction effect was statistically significant (P-value varying from 0.04 to 0.005$)$, indicating that these QTLs were segregating in the founder lines.

Half-sib analysis

Quantitative trait loci analysis using the paternal half-sib model was first performed across families. As QTLs for some traits were segregating in only one family, within-family analyses were also carried out to find the best positions and effects of identified QTLs.

Half-sib analyses revealed suggestive QTLs for Adj_WG35-41 (283 cM, between LEI0169 and LEI0106) and Adj_FC35-41 (317 cM, between LEI0106 and ADL0183) in family 1 and Adj_heart weight (99 cM between LEI0146 and LEI0174) in family 3. This analysis also revealed significant QTLs ( $P<0.05$ genome-wide) for Adj_gizzard (372 cM between MCW0020 and ROS0025) in family 3, Adj_liver (170 cM between LEI0138 and MCW0068) in 
family 2, and Adj_lungs (97 cM between LEI0146 and LEI0174) in family 1. These QTLs were not detected in the line-cross analysis; a probable hypothesis is that the QTL alleles for these traits may still be segregating in the lines. However, some QTLs detected in the line-cross analyses were not detected using the half-sib model. These differences might be explained in part by the dominant nature of some QTLs detected with the line-cross model (Table 1$)$. For instance, QTLs affecting Adj_wings (28 cM), Adj_abdominal fat (251 cM) and Adj_feet (194 cM) showed dominance effects (Table 1) and were not detected under the half-sib model. Dominance effects contribute little to the allele substitution effect estimated in the half-sib analysis (De Koning et al. 1999).

Figure 2 shows QTLs for BW42, and several carcass traits mapped to the same region in family 1 , suggesting that the same gene or a group of linked genes is involved in the phenotypic variation of those traits. An interesting QTL for Adj_FC35-41 was mapped at 317 cM (Fig. 2). This QTL was identified using BW42 as a covariate, suggesting that it is not affected by the body weight QTL. This region may be a good candidate for fine-mapping studies and multi-trait analyses to confirm if this QTL affects FC35-41 independent of body weight.

This is the first study to show QTLs mapped for feed conversion, liver, gizzard, lungs, heart and feet weights, and intestine length. We also mapped QTLs to regions already identified in other populations for body weight, weight gain, feed intake, wings weight, drums and thighs weight, and abdominal fat.

Quantitative trait loci mapped for Adj_FC35-41 and Adj_abdominal fat are of great importance for the poultry industry. Feed represents a major component of production costs. Measurement of feed conversion requires feed intake to be recorded individually for a large number of animals, thereby limiting direct selection. Today, consumers and consequently the industry seek reduced abdominal fat. As abdominal fat is positively correlated with body weight, selection for growth rate has been accompanied by increased abdominal fat. Because these QTLs were mapped after adjusting for body weight, genes distinct from those affecting growth may control part of the phenotypic variance of these traits. As selection for feed conversion and abdominal fat are difficult to perform using 
traditional methods, identified QTL regions should be investigated further in order to identify genes that could be used for MAS.

Quantitative trait loci mapped for Adj_heart and Adj_lungs in the half-sib analyses also indicate a candidate region for future studies. These results suggest that genes located in this region affect the weight of heart and lungs independent of the genes involved in body weight. Identification of genes of this nature could help reduce metabolic problems such as ascites, which causes great economic losses to the poultry industry.

The new population described herein allowed confirmation of several QTLs previously reported in other studies as well as identification of new QTLs. This new resource population is open to collaborations for studies of genes related to growth and carcass traits.

\section{Acknowledgements}

The authors thank the United States Poultry Genome Project (Dr Jerry Dodgson and Dr Hans H. Cheng) for primer donation. Financial support was provided by FAPESP (Fundac,ão de Amparo a' Pesquisa do Estado de Sao Paulo), EMBRAPA/PRODETAB, FUNCITEC and CNPq in Brazil, as well as BBSRC in the UK. LL Coutinho is a recipient of a research productivity scholarship from CNPq. 


\section{References}

Burt D.W. (2002) Applications of biotechnology in the poultry industry. World's Poultry Science Journal 58, 5-13.

Churchill G.A. \& Doerge R.W. (1994) Empirical threshold values for quantitative trait mapping. Genetics 138, 963-71.

De Koning D.J., Janss L.L.G., Rattink A.P., Van Oers P.A.M., de Vries B.J., Groenen M.A.M., Van der Poel J.J., de Groot P.N., Brascamp E.W. \& Van Arendonk J.A.M. (1999) Detection of quantitative trait loci for backfat thickness and intramuscular fat content in pigs (Sus scrofa). Genetics 152, 1679-90.

De Koning D.J., Haley C.S., Windsor D., Hocking P.M., Griffin H., Morris A., Vincent J. \& Burt D.W. (2004) Segregation of QTL for production traits in commercial meat-type chickens. Genetical Research 83, 211-20.

Green P., Falls K. \& Crooks S. (1990) Program CRI-MAP, Version 2.4. Washington University School of Medicine, St Louis. Groenen M.A.M., Cheng H.H., Bumstead N. et al. 2000. A consensus linkage map of the chicken genome. Genome Research 10, 137-47.

Haley C.S., Knott S.A. \& Elsen J.M. (1994) Mapping quantitative trait loci in crosses between outbred lines using least squares. Genetics 136, 1195-207.

Ikeobi C.O.N., Woolliams J.A., Morrice D.R., Law A., Windsor D., Burt D.W. \& Hocking P.M. (2002) Quantitative trait loci affecting fatness in the chicken. Animal Genetics 33, 428-35.

Ikeobi C.O.N., Woolliams J.A., Morrice D.R., Law A., Windsor D., Burt D.W. \& Hocking P.M. (2004) Quantitative trait loci for meat yield and muscle distribution in a broiler layer cross. Livestock Production Science 87, 143-51.

International Chicken Genome Sequencing Consortium (2004) Sequence and comparative analysis of the chicken genome provide unique perspectives on vertebrate evolution. Nature $432,695-716$. 
Jennen D.G.J., Vereijken A.L.J., Bovenhuis H., Crooijmans R.P.M.A., Veenendaal A., der Poel J.J. \& Groenen M.A.M. (2004) Detection and localization of quantitative trait loci affecting fatness in broilers. Poultry Science 83, 295-301.

Knott S.A., Elsen J.M. \& Haley C.S. (1996) Methods for multiplemarker mapping of quantitative trait loci in half-sib populations. Theoretical and Applied Genetics 93, 71-80.

Knott S.A., Marklund L., Haley C.A.S. et al. (1998) Multiple marker mapping of quantitative trait loci in a cross between outbred wild boar and large white pigs. Genetics 149, 1069-80.

Lander E. \& Kruglyak L. (1995) Genetic dissection of complex traits: guidelines for interpreting and reporting linkage results. Nature Genetics 11, 241-7.

Ledur M.C., Zanella E.L., Schmidt G.S., Jaenisch F.R.F., Saatkamp M.G., Bassi L.J. \& Coutinho L.L. (2000a) Peso e características de carcac,a em linhagens utilizadas no desenvolvimento de populacoes referencia para deteccao de QTL em aves. Revista Brasileira7 de Ciencia Avıcola 2 (Suppl. 2), 73.

Ledur M.C., Zanella E.L., Schmidt G.S., Jaenisch F.R.F., Silva V. S., Ventura L. \& Coutinho L.L. (2000b) Divergence of Strains and Strain Crosses used to Develop New Reference Populations for QTL Studies in Poultry. Proceedings of the XXI World's Poultry Congress, Montreal, Canada. CD Rom: abstracts/aug22/LEDUR_1.doc.

Marklund L., Nystrom P., Stern S., Andersson-Eklund L. \& Andersson L. (1999) Confirmed quantitative trait loci for fatness and growth on pig chromosome 4. Heredity 82, 134-41.

Nones K., Ledur M.C., Ruy D.C., Baron E.E., Moura A.S.A.M.T. \& Coutinho L.L. (2005) Genetic linkage map of chicken chromosome 1 from a Brazilian resource population. Scientia Agricola 62, 12-7.

Seaton G., Haley C.S., Knott S.A., Kearsey M. \& Visscher P.M. (2002) QTL express: mapping quantitative trait loci in simple and complex pedigrees. Bioinformatics 18, 339-40.

Sewalem A., Morrice D. M., Law A., Windsor D., Haley C. S., Ikeobi C. O. N., Burt D. W. \& Hocking P. M. (2002) Mapping of quantitative trait loci for body weight at three, six and nine weeks of age in a broiler layer cross. Poultry Science 81, 1775-81. 
Spelman R.J. \& Bovenhuis H. (1998) Moving from QTL experimental results to the utilization of QTL in breeding programmes. Animal Genetics 29, 77-84.

Tatsuda K. \& Fujinaka K. (2001) Genetic mapping of the QTL affecting body weight in chickens using a F2 family. British Poultry Science 42, 333-7.

Van Kaam J.B.C.H.M., Groenen M.A.M., Bovenhuis H., Veenendaal A., Vereijken A.L.J. \& Van Arendonk J.A.M. (1999a) Whole genome scan in chickens for quantitative trait loci affecting growth and feed efficiency. Poultry Science 78, 15-23.

Van Kaam J.B.C.H.M., Groenen M.A.M., Bovenhuis H., Veenendaal A., Vereijken A.L.J. \& Van Arendonk J.A.M. (1999b) Whole genome scan in chickens for quantitative trait loci affecting carcass traits. Poultry Science 78, 1091-9. 
Fig.1

Markers tested in selective genotyping of chromosome $1 .{ }^{*}$ The horizontal line indicates significant association $(P<0.10)$ with body weight at 42 days adjusted for hatch and sex. Marker positions are in accordance with one or more of the maps (Compton, East Lansing, Wageningen or consensus) that are available at http://www.animalgenome.org/ 8 chickmap.

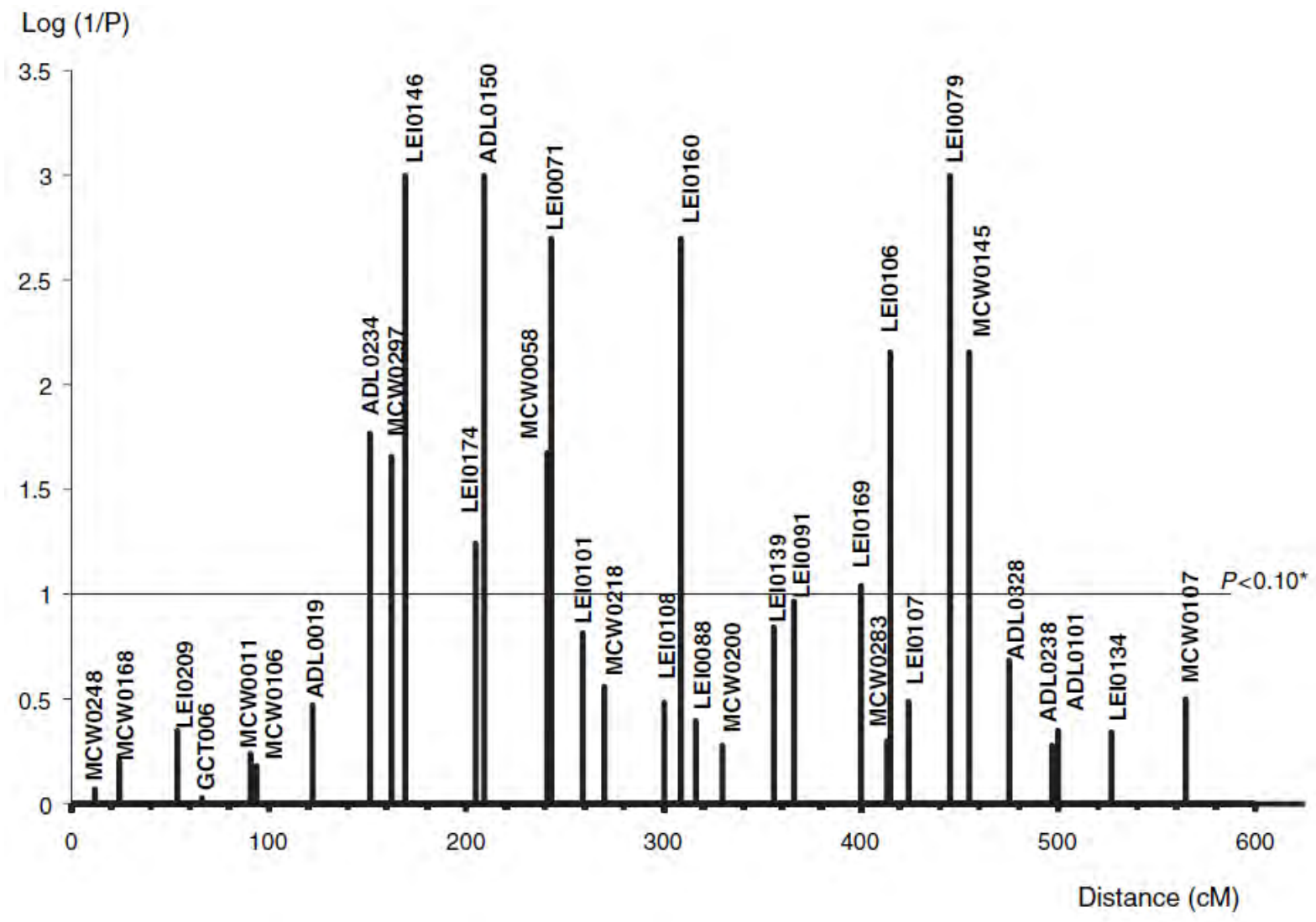


Fig.2

Test statistic values from the Chromosome 1 quantitative trait loci mapping analysis of body weight at 42 days (BW42), heart, drums and thighs, breast, and carcass weights and feed conversion between days 35 and 41 adjusted for body weight at 35 days (Adj_FC35-41) for family 1 using a withinfamily paternal half-sib model. Arrows indicate marker positions.

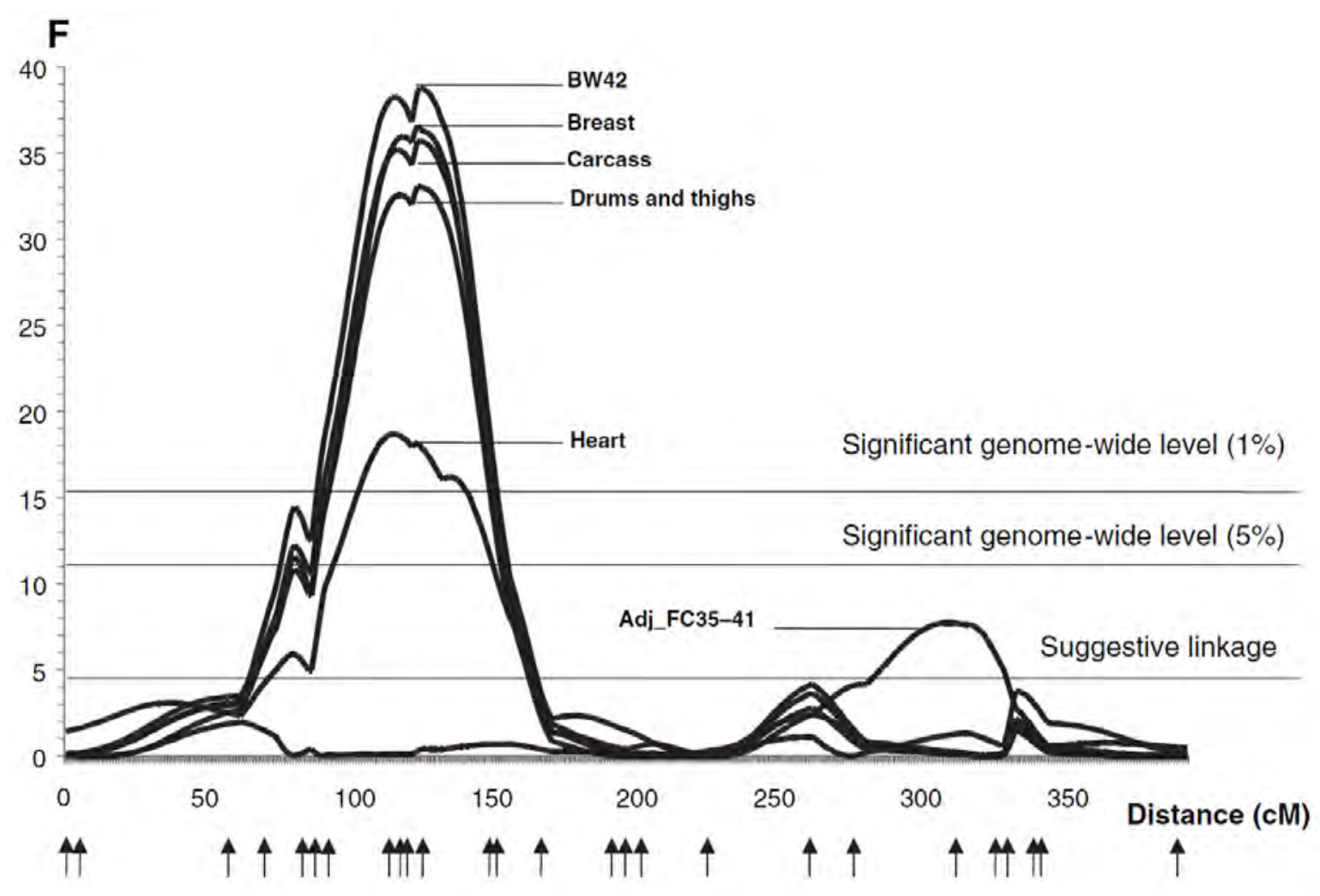


Tab.1

Quantitative trait loci (QTL) mapped in the line-cross analysis on chromosome 1.

\begin{tabular}{|c|c|c|c|c|c|c|}
\hline Position $(\mathrm{cM})^{1}$ & Trait & Flanking markers & F-ratio & $\begin{array}{l}\text { Additive } \\
\text { effect (SE) }\end{array}$ & $\begin{array}{l}\text { Dominance } \\
\text { effect (SE) }\end{array}$ & $\begin{array}{l}\text { Phenotypic } \\
\text { variance }(\%)^{3}\end{array}$ \\
\hline 28 & Adj_wings weight $(\mathrm{g})^{2}$ & MCW0208-ADL0188 & $5.23^{4}$ & $0.60(0.36)$ & $-2.35(0.83)$ & 1.35 \\
\hline 53 & Lungs weight (g) & MCW0208-ADL0188 & $8.20^{4}$ & $-0.40(0.21)$ & $-1.04(0.37)$ & 1.67 \\
\hline 81 & Body weight at 35 days (g) & LEI0068-MCW0297 & $10.41^{6}$ & $29.31(6.66)$ & $7.41(9.46)$ & 2.94 \\
\hline 81 & Body weight at 42 days (g) & LEI0068-MCW0297 & $8.54^{5}$ & $24.57(8.48)$ & $15.19(12.05)$ & 1.35 \\
\hline 82 & Carcass weight (g) & LEI0068-MCW0297 & $8.65^{5}$ & $24.57(8.48)$ & $15.19(12.05)$ & 1.35 \\
\hline 83 & Intestine length $(\mathrm{cm})$ & LEI0068-MCW0297 & $11.12^{6}$ & $3.04(0.72)$ & $-0.30(1.05)$ & 2.51 \\
\hline 84 & Adj_intestine length $(\mathrm{cm})^{2}$ & LEI0068-MCW0297 & $6.98^{4}$ & $1.70(0.64)$ & $-0.95(0.92)$ & 0.98 \\
\hline 84 & Wings weight (g) & LEI0068-MCW0297 & $8.54^{5}$ & $2.51(0.64)$ & $0.94(0.93)$ & 2.28 \\
\hline 86 & Liver weight (g) & MCW0297-LEI0146 & $7.67^{4}$ & $0.90(0.25)$ & $0.54(0.36)$ & 2.10 \\
\hline 88 & Lungs weight (g) & MCW0297-LEI0146 & $10.63^{5}$ & $0.72(0.17)$ & $0.61(0.20)$ & 4.04 \\
\hline 88 & Feet weight $(\mathrm{g})$ & MCW0297-LEI0146 & $10.62^{6}$ & $1.52(0.35)$ & $0.39(0.49)$ & 2.83 \\
\hline 93 & Residual carcass weight (g) & LEI0146-LEI0174 & $8.78^{5}$ & $6.87(1.79)$ & $3.38(2.62)$ & 2.30 \\
\hline 94 & Adj_abdominal fat weight $(\mathrm{g})^{2}$ & LEI0146-LEI0174 & $12.18^{6}$ & $-1.34(0.28)$ & $0.32(0.41)$ & 3.44 \\
\hline 97 & Drums and thighs weights (g) & LEI0146-LEI0174 & $8.06^{4}$ & $8.58(2.21)$ & $3.29(3.47)$ & 2.22 \\
\hline 100 & Heart weight (g) & LEI0146-LEI0174 & $11.33^{6}$ & $0.39(0.08)$ & $0.02(0.14)$ & 3.09 \\
\hline 125 & Feed intake from 35 to 41 days (g) & ADL0150-ADL0319 & $6.15^{4}$ & $18.08(5.86)$ & $-14.87(8.31)$ & 1.63 \\
\hline 138 & Body weight at 42 days (g) & ADL0319-MCW0058 & $8.04^{4}$ & $32.14(9.26)$ & $-12.99(15.0)$ & 1.66 \\
\hline 138 & Breast weight (g) & ADL0319-MCW0058 & $9.05^{5}$ & $7.54(1.75)$ & $-3.35(2.84)$ & 2.73 \\
\hline 139 & Carcass weight $(\mathrm{g})$ & ADL0319-MCW0058 & $7.49^{4}$ & $23.55(6.55)$ & $-8.86(10.61)$ & 1.79 \\
\hline 184 & Body weight at 35 days (g) & LEI0138-MCW0068 & $8.15^{4}$ & $23.50(6.96)$ & $-2.26(11.50)$ & 1.51 \\
\hline 194 & Adj_feet weight $(\mathrm{g})^{2}$ & MCW0068-ADL0020 & $7.25^{4}$ & $-0.53(0.16)$ & $0.55(0.24)$ & 1.98 \\
\hline 196 & Abdominal fat weight (g) & ADL0020-LEI0160 & $6.11^{4}$ & $0.98(0.35)$ & $-1.27(0.52)$ & 1.62 \\
\hline 200 & Residual carcass weight (g) & ADL0020-LEI0160 & $7.20^{4}$ & $4.85(1.71)$ & $-5.13(2.67)$ & 1.45 \\
\hline 251 & Adj_abdominal fat weight $(\mathrm{g})^{2}$ & ADL0148-MCW036 & $7.90^{4}$ & $0.66(0.32)$ & $-0.97(0.57)$ & 0.81 \\
\hline 332 & Body weight at 35 days (g) & LEI0079-MCW0145 & $7.15^{4}$ & $26.70(6.34)$ & $-7.35(9.51)$ & 2.56 \\
\hline 332 & Body weight at 42 days (g) & LEI0079-MCW0145 & $7.15^{4}$ & 30.91 (7.99) & $-7.55(11.85)$ & 2.10 \\
\hline 332 & Carcass weight (g) & LEI0079-MCW0145 & $5.61^{4}$ & $19.78(5.65)$ & $-5.03(8.38)$ & 1.68 \\
\hline 332 & Gizzard weight (g) & LEI0079-MCW0145 & $6.72^{4}$ & $0.75(0.21)$ & $-0.19(0.31)$ & 1.80 \\
\hline 332 & Heart weight (g) & LEI0079-MCW0145 & $7.99^{4}$ & $0.21(0.07)$ & $-0.10(0.11)$ & 1.13 \\
\hline 332 & Feet weight (g) & LEI0079-MCW0145 & $8.64^{5}$ & $1.22(0.34)$ & $-0.18(0.50)$ & 1.74 \\
\hline 333 & Breast weight (g) & LEI0079-MCW0145 & $7.29^{4}$ & $5.12(1.61)$ & $-2.19(2.40)$ & 1.39 \\
\hline 334 & Wings weight (g) & LEI0079-MCW0145 & $7.81^{4}$ & $2.35(0.63)$ & $-0.51(0.94)$ & 1.95 \\
\hline 339 & Intestine length $(\mathrm{cm})$ & LEI0079-MCW0145 & $12.92^{6}$ & $3.49(0.68)$ & $0.06(1.00)$ & 3.78 \\
\hline 373 & Adj_intestine length $(\mathrm{cm})^{2}$ & MCW0020-ROS0025 & $9.80^{6}$ & $3.87(0.89)$ & $-1.89(1.96)$ & 2.80 \\
\hline
\end{tabular}

${ }^{1}$ Position of QTL relative to the first marker in the set on chromosome 1. MCW10 is at 72 cM on the consensus map.

${ }^{2}$ Quantitative trait loci analyses include BW35 or BW42 as a covariate in the model.

${ }^{3}$ Proportional decrease in the residual sums of squares by fitting the model with the QTL compared with the reduced model.

${ }^{4}$ Suggestive linkage.

${ }^{5}$ Significant linkage at $\mathrm{P}<0.05$ genome-wide.

${ }^{6}$ Significant linkage at $\mathrm{P}<0.01$ genome-wide. 